\title{
The Quota-Based Compensation Plan in Fashion Retailing Industry under Asymmetric Information
}

\author{
Mingzhu Yu, ${ }^{1}$ Ruina Yang, ${ }^{2}$ and Lijun $\mathrm{Ma}^{3}$ \\ ${ }^{1}$ Department of Transportation Engineering, College of Civil Engineering, Shenzhen University, Shenzhen 518060, China \\ ${ }^{2}$ Department of Management Science, School of Management, Xian Jiaotong University, Xian 710049, China \\ ${ }^{3}$ Department of Management Science, College of Management, Shenzhen University, Shenzhen 518060, China
}

Correspondence should be addressed to Ruina Yang; rnyang@mail.xjtu.edu.cn

Received 20 March 2014; Revised 2 May 2014; Accepted 13 May 2014; Published 1 June 2014

Academic Editor: Pui-Sze Chow

Copyright (C) 2014 Mingzhu Yu et al. This is an open access article distributed under the Creative Commons Attribution License, which permits unrestricted use, distribution, and reproduction in any medium, provided the original work is properly cited.

\begin{abstract}
We study a compensation plan problem in the fashion retailing industry, which involves a risk-neutral fashion retailer and a riskneutral salesperson, in a two-stage game framework with asymmetric information. In the first stage, the fashion retailer provides a menu of compensation plans to the salesperson who decides which plan to sign based on his superior market demand information. Confronted with the asymmetric demand information, the fashion retailer could observe market information from the salesperson's response by designing a menu of compensation plans rather than a single one to the salesperson. In the second stage, the fashion retailer then makes production decision and the salesperson determines his selling effort. We consider both adverse selection and moral hazard. We adopt the quota-based plan to derive the fashion retailer's optimal compensation plan design and the salesperson's best response. We emphasize the impact of the quota level on the system outcomes. The results reveal that a higher quota level is disadvantageous to the fashion retailer but advantageous to the salespersons.
\end{abstract}

\section{Introduction}

The information, especially the demand information, is particularly valuable for the fashion supply chain. Large fashion companies (such as deluxe luxury brands Gucci and Burberry) often employ a sales force which is comprised of multiple salespersons. These salespersons are often called fashion sales representatives. In the sales compensation problem, the sales force employed by a fashion retailer may have an advantage over the fashion retailer in access to the market information. The sales force usually has some demand data directly related to the market and could do analysis to dig some information. This superior market information could be exploited by the salesperson to assess the demand potential, determine selling effort, and choose the compensation plan from the fashion retailer. If the market information is revealed, the fashion retailer could make better production plan, such as deciding the clothing color, the raw material quality, the style, and the quantity. This asymmetric information situation is referred to as "adverse selection" in the agency theory (see, e.g., Kreps [1]). Another issue in the compensation problem is the so-called "moral hazard," which implies that the salesperson's selling effort, which directly determines the sales volume, is unobservable to the fashion retailer. Hence, the main concern of the fashion retailer is to design an appropriate rewarding scheme to induce the sales force to reveal truthful demand information and, at the same time, encourage them to work hard. We will consider both issues in this paper.

Specifically, we consider the compensation problem involving one fashion retailer and one salesperson. Both the fashion retailer and the salesperson are risk-neutral. It is widely accepted in the literature that a typical way out of the compensation problem combining moral hazard with adverse selection is to offer a menu of contracts to the salespersons. This approach provides a mechanism to avoid the distortion of the private information, which is the main objective of our paper. At first, the fashion retailer as a leader provides quota-based compensation plan bundles to the salesperson and intends to learn something about 
the demand information from the salesperson's choice. The quota-based compensation plan includes a fixed salary and a variable income which is proportional to the sales volume above a prespecified quota. Then, the salesperson as a follower privately observes the market status and decides which plan to sign. Finally, the sales volume is realized and the corresponding payment is made by the fashion retailer to the salesperson.

We employ a two-stage game to address the design of compensation plan of the fashion retailer and the response of the salesperson. We firstly analyze the salesperson's best response to the compensation plan signed in the first stage. Next we employ the screening model to formulate the fashion retailer's optimal design of compensation scheme. We fully characterize the optimal parameters of the commission rate and the fixed salary under the quota-based compensation plan. Our paper contributes to the literature by considering both the asymmetric information between the sales force and the fashion retailer and the impact of the quota level on their optimal strategies.

The rest of this paper is organized as follows. In Section 2 we review the related literature. In Section 3 we carry out the model framework. In Section 4 we study the salesperson's optimal response and in Section 5 we study the fashion retailer's compensation plan design. We investigate the impact of the specified quota on the optimal strategies of the fashion retailer as well as the salespersons in Section 6. Finally, we conclude the whole paper with managerial insights in Section 7. All proofs are presented in the appendix.

\section{Literature Review}

There are three streams of studies that are related to our research: the sales force management, quota-based compensation plan, and the contracting theory in the fashion supply chains.

In the literature, there exists extensive research for sales force management. We refer to Coughlan and Sen [2] and Coughlan [3] for comprehensive reviews. Existing research about the sales force compensation involves two kinds of relationship between the selling effort and the sales volume: the deterministic relationship (refer to Farley [4], Tapiero and Farley [5], Weinberg [6], etc.) and the stochastic relationship. The latter means that the sales volume is a stochastic function of the salesperson's selling effort which is unobservable to the fashion retailer. Basu et al. [7] apply agency theory for the first time to model the sales force compensation problem. They maximize the fashion retailer's profit and take into account the likely behavior of the salesperson in response to the compensation plan. Their research is then further studied by Srinivasan [8], Coughlan and Sen [2], Lal and Srinivasan [9], and so forth. After that, the basic model of one product, one salesperson and symmetric information is extended by Lal [10], Lal and Staelin [11], Dearden and Lilien [12], Rao [13], Lal and Srinivasan [9], and Chen [14, 15] to allow for multiple products, multiple salespersons, asymmetric information, and so on. Chen [14-16] studies the problem of sales force compensation in a setting of one fashion retailer and one salesperson by considering the impact of sales force behavior on a fashion retailer's production and inventory system. Porteus and Whang [17] present a specific connection with sales force incentives and manufacturing incentives. Their model is single-period and thus ignores the dynamic nature of sales force behavior. In our paper, we study the compensation plan problem involving one fashion retailer and one salesperson with both moral hazard and adverse selection. Our research contributes to the literature by considering the fashion retailer's design of compensation plan to a salesperson by anticipating that a production decision has to be made after contract signing, but before sales realization.

In terms of the literature on the quota-based compensation plan, our analysis is different from those that are studied before. Rao [13] and Mantrala et al. [18] propose a quota-based plan, which is linear in the sense that sales below quota would lead to a reduction in compensation at the same rate as the incremental compensation paid to sales above quota. Lal and Staelin [11] study a menu of quotabased compensation plans in the presence of information asymmetry between the fashion retailer and the salesperson. Mantrala et al. [18] investigate how the quota can serve as a fashion retailer's mechanism to learn about the selling effectiveness of the salesperson. Raju and Srinivasan [19] consider a basic quota-based compensation plan where the fixed salary and commission rate are fixed across the entire sales force. We highlight the formulation of the quota-based compensation with one salesperson. In addition, our analysis contributes to the literature in considering the asymmetric information and providing interesting insights into how the specified quota affects the fashion retailer and salesperson's optimal strategies.

Some research is found in the contracting theory of fashion supply chain. Chiu et al. [20] consider the effect of the sales effort on the sales rebate contract used in the fashion supply chains. Kurata and Yue [21] study the scan-back trade promotion mode which enables information sharing between the manufacturer and retailer of the fashion industry. Xu et al. [22] investigate different coordinating contracts (coordinating revenue sharing contract, two-part tariff contract, sales rebate and penalty contract, and revenue sharing with twopart tariff contract) in the fashion supply chain with a riskaverse retailer. Wang et al. [23] study two kinds of outsourcing structure under two different contracts which could be widely used in the fashion industry. Our paper focuses on the sales force management problem between the fashion retailer and the employed salesperson. We mainly study the design and the effect of the quota-based compensation plan.

\section{Model Framework}

3.1. Sales Volume. We propose a model in which a fashion retailer (she/her) employs a salesperson (he/him) to sell a product. Let $e$, a nonnegative real number, be the effort level of the salesperson. Denote the effectiveness of the selling effort by $\mu$. We assume that the salesperson's total sales volume is

$$
D=d+\mu e+\varepsilon
$$




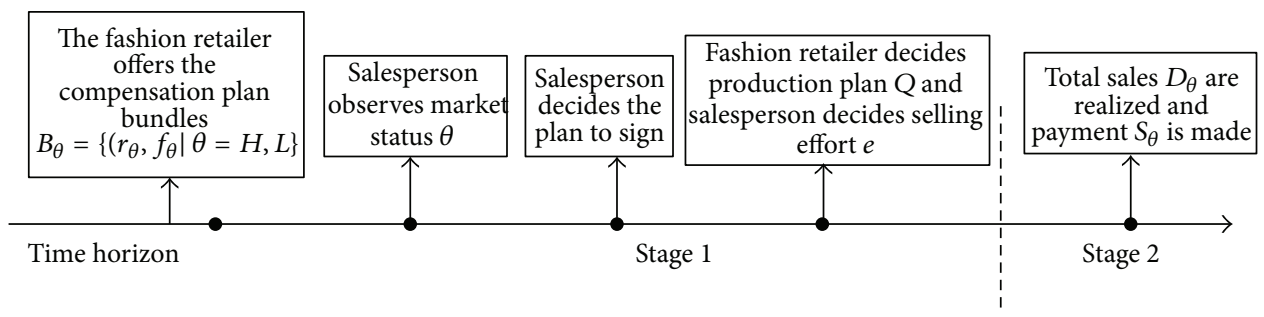

FIgURE 1: The event timeline.

Here, $d$ is a random variable denoting the market status that the salesperson faces. Assume the market status, which is private information of the salesperson, has two states, high and low, as follows:

$$
d= \begin{cases}d_{H}, & \text { with probability } \rho ; \\ d_{L}, & \text { with probability } 1-\rho .\end{cases}
$$

Here, $d_{H}$ and $d_{L}$ correspond to the high and low states of market status, respectively, and $d_{H}>d_{L}>0$. Assume that $d_{L}$ is sufficiently large that the probability of $D$ being negative is negligible. This kind of two-state assumption is widely used in the literature and is sufficient to capture the main features of compensation plan (refer to Chen [14], Ha and Tong [24], $\mathrm{Li}$ et al. [25], and Lee and Yang [26]). We denote the state by $\theta$, $\theta=H$ or $L$, which is observed by the salespersons but not the fashion retailer. Moreover, $\varepsilon$ is a random variable independent of $d$, representing a random noise with $\varepsilon \sim U[-Z, Z]$, where $Z$ is a nonnegative real number.

3.2. The Quota-Based Contract. Facing the existence of moral hazard and adverse selection, the fashion retailer's typical solution is to offer compensation plan bundles to the salesperson. In our model, due to the two-point distribution of state, the fashion retailer provides two alternative bundles to the salesperson $B_{\theta}=\left\{\left(r_{\theta}, f_{\theta}\right) \mid \theta=H, L\right\}$, where $r_{\theta}$ is the commission rate and $f_{\theta}$ is the fixed salary. We let $S_{\theta}$ be the total payment made to the salesperson. We study a quota-based compensation plan. Since the fashion retailer cannot directly observe the salesperson's selling effort level, she compensates him based on his realized value of $D$. Hence, the total payment to the salesperson based on $D_{\theta}$ is

$$
S_{\theta}\left(D_{\theta}\right)=f_{\theta}+r_{\theta}\left(D_{\theta}-q\right)^{+}
$$

where $q$ is the prespecified quota, an exogenous constant, and $q \in[q, \bar{q}]$.

The payment function of the quota-based plan is a fixed salary plus a commission income if the sales volume exceeds a prespecified quota. For easy implementation, we consider a quota-based plan, where the quota $q$ is given or fixed across a geographic area.

3.3. Sequence of Events. The relationship between the fashion retailer and the salesperson is like that of a principal and a sales agent. The latter sells the product on behalf of the former. The salesperson's sales volume increases when he puts more selling effort, which is not observable to the fashion retailer. The fashion retailer designs the compensation plan and makes her production decision. We assume that both the fashion retailer and the salesperson are risk-neutral.

We model the compensation plan problem under asymmetric information as a two-stage game. The sequence of events is presented in Figure 1 and described as follows.

Stage 1. (1) The fashion retailer offers a menu of compensation plans to the salesperson. (2) The salesperson privately observes the market status. (3) The salesperson decides whether or not to accept the compensation plan and, if so, which plan to sign. (4) With the signed compensation plan, the fashion retailer determines the production quantity, and the salesperson determines how much effort to exert.

Stage 2. The uncertainty of demand is resolved. Both the fashion retailer and the salesperson observe the total sales. At the same time, the associated payments depending on the compensation plan signed are made from the fashion retailer to the salesperson.

3.4. Production Plan. According to the above defined sequence of events, the fashion retailer needs to determine the production quantity before the total sale is realized. Let $Q$ be the production quantity and let $c$ be the production cost. If $Q \geq D$, the excess products are salvaged at $v$ per unit. On the other hand, if $Q<D$, the excess demand must be satisfied via an emergency supply such as a special production run at a cost of $c^{\prime}$ per unit, which is usually greater than $c$. Let $p$ denote the selling price of the product. To avoid trivial case, we assume $v<c<c^{\prime}<p$. The fashion retailer's production cost is

$$
P(Q \mid D)=c Q-v(Q-D)^{+}+c^{\prime}(D-Q)^{+} .
$$

Below we use backward induction to address the above defined compensation plan problem. We firstly consider the salesperson's optimal decision when he is offered a bundle of compensation plans in the first stage. Then we study the fashion retailer's optimal design of plans by taking the salesperson's best response into consideration.

\section{Salesperson's Best Response}

Given the bundles of compensation plans, the salesperson privately observes the market status. Then he decides which plan to sign and how much selling efforts to exert. Assume 
the salesperson is risk-neutral and his disutility function is $V(e)=(1 / 2) e^{2}$, an increasing convex function. The objective of the salesperson is to maximize the expected net income, the wage $S(D)$ minus the disutility function $V(e)$. Suppose he chooses the bundle $B_{\tau}, \tau=H$ or $L$, and when the state is $\theta, \theta=H$ or $L$, the salesperson's problem is to maximize the expected net income $\pi_{\theta}\left(B_{\tau}\right)$ as follows:

$$
\begin{aligned}
\pi_{\theta}\left(B_{\tau}\right) & =\max _{e}\left\{E\left[S_{\tau}\left(D_{\theta}\right)-V(e)\right]\right\} \\
& =\max _{e}\left\{E\left[f_{\tau}+r_{\tau}\left(D_{\theta}-q\right)^{+}-\frac{1}{2} e^{2}\right]\right\} .
\end{aligned}
$$

We derive the salesperson's best response in Lemma 1.

Lemma 1. The salesperson's optimal response of selling effort under the bundle $B_{\tau}$ is determined by

$$
e^{*}\left(B_{\tau}\right)=\frac{r_{\tau} \mu\left(d_{\theta}+Z-q\right)}{2 Z-r_{\tau} \mu^{2}} .
$$

Here, $Z$ denotes the random noise, and the term $d_{\theta}+Z$ represents the base level of potential sales when the state is $\theta$. The term $d_{\theta}+Z-q$ is the difference between the base potential sales and the quota specified in a geographic area. Lemma 1 indicates that the optimal effort level increases with $d_{\theta}+Z-q$ but decreases with $q$. Namely, the salesperson will work harder if the market implies a higher level of base potential sales but a lower level of prespecified quota. For example, in the fashion industry market, the fashion sales representatives will work harder in the peak season or with a lower level of quota provided by the fashion company.

Lemma 1 also implies that the optimal effort level enhances with the commission rate $r_{\tau}$, but the fixed salary $f_{\tau}$ does not affect the optimal effort. Hence, the fashion retailer should choose a higher commission rate to stimulate hard work of the fashion sales representatives.

\section{Optimal Compensation Plan}

We now analyze the design of compensation plan for the fashion retailer in the presence of asymmetric information. Since the market state is unobservable to the fashion retailer, the fashion retailer offers two bundles $B_{\theta}=\left\{\left(r_{\theta}, f_{\theta}\right) \mid \theta=\right.$ $H, L\}$ to the salesperson.

As the production decision is made before the sales realization, the fashion retailer's total profit $F$ is given by

$$
\begin{aligned}
F= & p D-S(D)-E\left[P_{i}(Q \mid D)\right] \\
= & (p-c) D-S(D)-c E[(Q-D)]+v E\left[(Q-D)^{+}\right] \\
& -c^{\prime} E\left[(D-Q)^{+}\right] .
\end{aligned}
$$

Obviously, both the first and the second terms of the fashion retailer's total profit $F$ do not depend on the production quantity $Q$. In this situation, the optimal production quantity $Q^{*}$ minimizes

$$
c E[(Q-D)]-v E\left[(Q-D)^{+}\right]+c^{\prime} E\left[(D-Q)^{+}\right] .
$$

If the total sales volume $D$ is realized, the optimal production quantity $Q^{*}$ can be obtained via the above standard Newsvendor problem. In this situation, the optimal production quantity $Q^{*}$ is determined by

$$
G\left(Q^{*}\right)=\frac{c^{\prime}-c}{c^{\prime}-v}
$$

where $G(\cdot)$ is the distribution function of $d$.

From the above analysis, we get that the determination of the production quantity may be separated from the design of optimal compensation plan. Additionally, the fashion retailer aims to design an appropriate compensation scheme so as to induce truth telling about the market status as well as motivate the salespersons to work hard. An optimal compensation plan can be derived by the following problem:

$$
\begin{array}{ll}
\max _{B_{H}(\cdot), B_{L}(\cdot)} & \rho\left[(p-c) D_{H}-S_{H}\left(D_{H}\right)\right] \\
& +(1-\rho)\left[(p-c) D_{L}-S_{L}\left(D_{L}\right)\right] \\
\text { s.t. } \quad & \left\{\begin{array}{l}
e_{L}^{*}=e\left(B_{L}(\cdot), L\right), \\
e_{H}^{*}=e\left(B_{H}(\cdot), H\right), \\
\pi_{L}\left(B_{L}(\cdot)\right) \geq \pi_{\min }, \\
\pi_{H}\left(B_{H}(\cdot)\right) \geq \pi_{\min }, \\
\pi_{L}\left(B_{L}(\cdot)\right) \geq \pi_{L}\left(B_{H}(\cdot)\right), \\
\pi_{H}\left(B_{H}(\cdot)\right) \geq \pi_{H}\left(B_{L}(\cdot)\right) .
\end{array}\right.
\end{array}
$$

The first two constraints are the incentive compatibility constraints for the salesperson's effort choices, the third and fourth constraints are the individual rationality constraints, which ensures the salesperson's minimum profit $\pi_{\min }$ in both high and low states, and the last two constraints guarantee that the salesperson maximizes his profit by truthfully revealing his information about the state.

Considering the salesperson's optimal effort level derived in the previous section, the fashion retailer's problem can be simplified as follows:

$$
\begin{array}{cl}
\max _{B_{H}(\cdot), B_{L}(\cdot)} \quad \begin{array}{l}
\rho \\
\\
\text { s.t. } \quad
\end{array} \quad\left\{\begin{array}{l}
\frac{\left.r_{L}\left(d_{L}+Z-q\right) D_{H}-S_{H}\left(D_{H}\right)\right]}{2\left(2 Z-r_{L} \mu^{2}\right)}+f_{L} \geq \pi_{\min }, \\
\frac{r_{H}\left(d_{H}+Z-q\right)^{2}}{2\left(2 Z-r_{H} \mu^{2}\right)}+f_{H} \geq \pi_{\min }, \\
\frac{r_{L}\left(d_{L}+Z-q\right)^{2}}{2\left(2 Z-r_{L} \mu^{2}\right)}+f_{L} \\
\geq \frac{r_{H}\left(d_{L}+Z-q\right)^{2}}{2\left(2 Z-r_{H} \mu^{2}\right)}+f_{H}, \\
\frac{r_{H}\left(d_{H}+Z-q\right)^{2}}{2\left(2 Z-r_{H} \mu^{2}\right)}+f_{H} \\
\geq \frac{r_{L}\left(d_{H}+Z-q\right)^{2}}{2\left(2 Z-r_{L} \mu^{2}\right)}+f_{L} .
\end{array}\right.
\end{array}
$$

Since $d_{H}>d_{L}$, we claim that the first and the last constraints are tight. By converting the constrained optimization problem into an unconstrained optimization problem, we derive the optimal parameters of a quota-based compensation plan in Theorem 2 . 
Theorem 2. The optimal parameters of a quota-based plan are given by the following.

(i) The optimal commission rate is as follows:

$$
\begin{gathered}
r_{L} \\
=\max \left\{0,\left(2 Z\left[2(1-\rho) \mu^{2}(p-c) T_{L}-\rho\left(T_{L}+T_{H}\right) I\right]\right)\right. \\
\times\left(2(1-\rho) \mu^{2} T_{L}^{2}\right. \\
\left.\left.+2(1-\rho) \mu^{4}(p-c) T_{L}-\rho \mu^{2}\left(T_{L}+T_{H}\right) I\right)^{-1}\right\} ; \\
r_{H}=\frac{2(p-c) Z}{T_{H}+\mu^{2}(p-c)} .
\end{gathered}
$$

(ii) And the optimal fixed salary is as follows:

$$
\begin{gathered}
f_{L}=\pi_{\min }-\frac{r_{L}\left(d_{L}+Z-q\right)^{2}}{2\left(2 Z-r_{L} \mu^{2}\right)} ; \\
f_{H}=f_{L}+\frac{r_{L}\left(d_{H}+Z-q\right)^{2}}{2\left(2 Z-r_{L} \mu^{2}\right)}-\frac{r_{H}\left(d_{H}+Z-q\right)^{2}}{2\left(2 Z-r_{H} \mu^{2}\right)} .
\end{gathered}
$$

Here, $T_{L}=d_{L}+Z-q, T_{H}=d_{H}+Z-q, T=T_{H}+T_{L}$, and $I=d_{H}-d_{L}$.

We define $I=d_{H}-d_{L}$ as the degree of information asymmetry of market status, and $p-c$ is the marginal profit of the product. By analyzing the first statement of Theorem 2, we get the following properties.

(1) The optimal commission rate in a low state $r_{L}$ is positive if and only if the term $(1-\rho) T_{L} / \rho T$ exceeds $I / 2 \mu^{2}(p-c)$.

(2) Both $r_{L}$ and $r_{H}$ are smaller than $2 Z / \mu^{2}$.

(3) $r_{H}>r_{L}$; namely, the optimal commission rate under a high state is higher than that in a low state; therefore, the fashion company should provide the fashion sales representatives with a higher commission rate in the peak season than in the low season.

(4) $r_{H}$ is independent of the probability of high state $\rho$.

(5) $r_{H}$ decreases with the market characteristics in a high state $T_{H}$ and increases with the marginal profit.

In the second statement of Theorem 2, we find the following.

(1) The optimal fixed salary in a low state $f_{L}$ decreases with $r_{L}$.

(2) $f_{H}$ increases with $r_{L}$ but decreases with $r_{H}$. Therefore, a lower fixed salary and a higher commission rate under a quota-based compensation plan will be valued by the fashion retailer.

\section{The Impact of the Quota}

As mentioned in our model, a quota-based compensation plan is easy to implement for a sales force employed by the fashion retailer. In the current paper, we propose a quotabased compensation plan where the quota is prespecified. However, if the fashion retailer is able to choose the quota of the quota-based plan, then what is the fashion retailer's best response in her strategy space? In this section we are going to analyze the impact of the quota value.

Note that we have derived the optimal parameters of commission rate and fixed salary under a quota-based plan under asymmetric information. Subsequently, in this section we begin with the sensitivity study of the impact of the specified quota on the optimal parameters. Finally, we implement numerical study to show the impact of the quota on the optimal strategies of the fashion retailer and the salesperson.

6.1. Impact of the Quota on the Optimal Parameters. From Theorem 2, we derive the optimal commission rate and fixed salary in both high and low states. Subsequently, we implement a sensitivity analysis to investigate the impact of the quota $q$ on the optimal commission rate as well as the fixed salary. The following lemma characterizes the sensitivity analysis of $q$.

Proposition 3 (sensitivity analysis). (i) The optimal commission rate in a high state $r_{H}$ is increasing in $q$ if and only if $\left((1-\rho)\left(d_{L}+Z-q\right) / \rho\left(d_{H}+Z-q\right)\right) \geq\left(\left(d_{H}-d_{L}\right) / \mu^{2}(p-c)\right)$. (ii) Moreover, if $(1-\rho)\left(d_{L}+Z-q\right) / \rho\left(d_{H}+Z-q\right)<\left(d_{H}-\right.$ $\left.d_{L}\right) / \mu^{2}(p-c)$, both $f_{L}$ and $f_{H}$ increase as $q$ rises.

Clearly, the condition $(1-\rho)\left(d_{L}+Z-q\right) / \rho\left(d_{H}+Z-q\right) \geq$ $\left(d_{H}-d_{L}\right) / \mu^{2}(p-c)$ can be interpreted as the proportion of the expected characteristics in a low state to the expected characteristics in a high state is no less than the proportion of information asymmetry for market status to the marginal profit multiplying $\mu^{2}$. Proposition 3 indicates that the fashion retailer should choose a higher commission rate but a lower fixed salary when given a larger quota $q$. As a higher level of quota $q$ implies worse characteristics for the territory, the fashion sales representative does not have the incentive to work hard for the fashion retailer.

6.2. The Impact of the Quota on the Optimal Strategies. This subsection presents the analysis of the impact of the quota specified in a geographic area on the optimal strategies of the fashion retailer and the salesperson. From Theorem 2, we can derive the optimal profits of the fashion retailer $\Pi^{0}$ as well as the salesperson $\pi^{0}$. Table 1 presents the fashion retailer and the salesperson's optimal profits $\left(\Pi^{0}\right.$ and $\left.\pi^{0}\right)$ under a quotabased compensation plan in both high and low states.

Subsequently, we implement numerical study to show the impact of the quota $q$ on the optimal profits of the fashion retailer as well as the salesperson. To guarantee that $d_{H}>d_{L}$, $p>c$, and $d_{L}-Z<q<d_{H}+Z$, we choose parameters as follows: $d_{H}=100, d_{L}=60, p=20, c=10, \mu=6$, and 
TABLE 1: Optimal profit under a quota-based compensation plan.

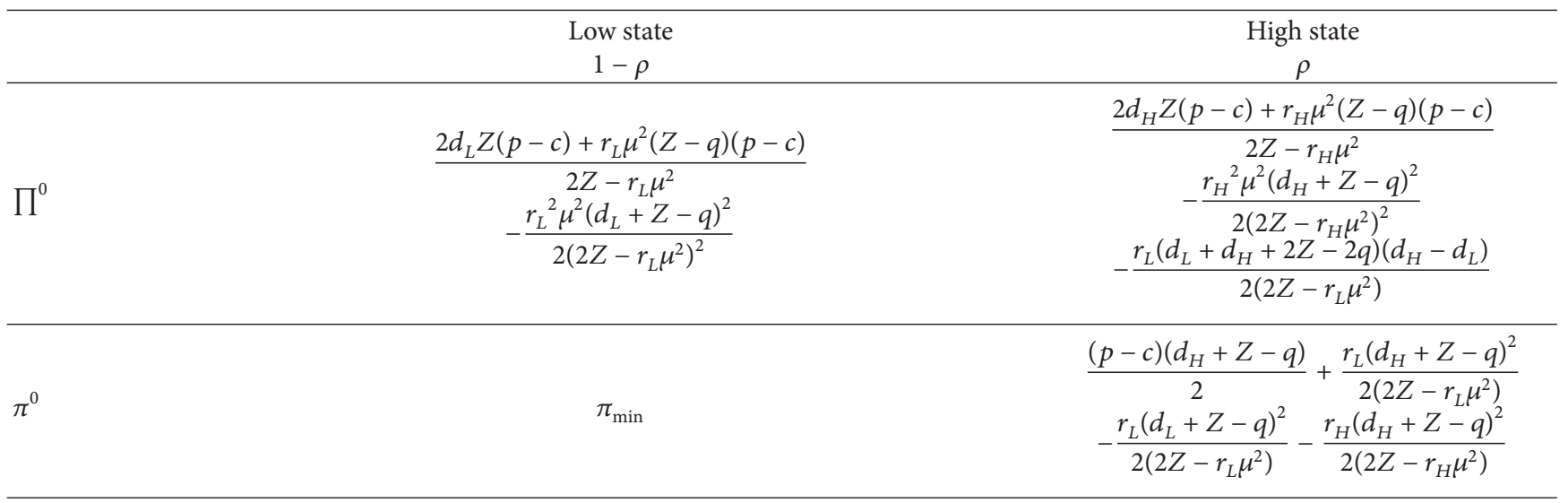

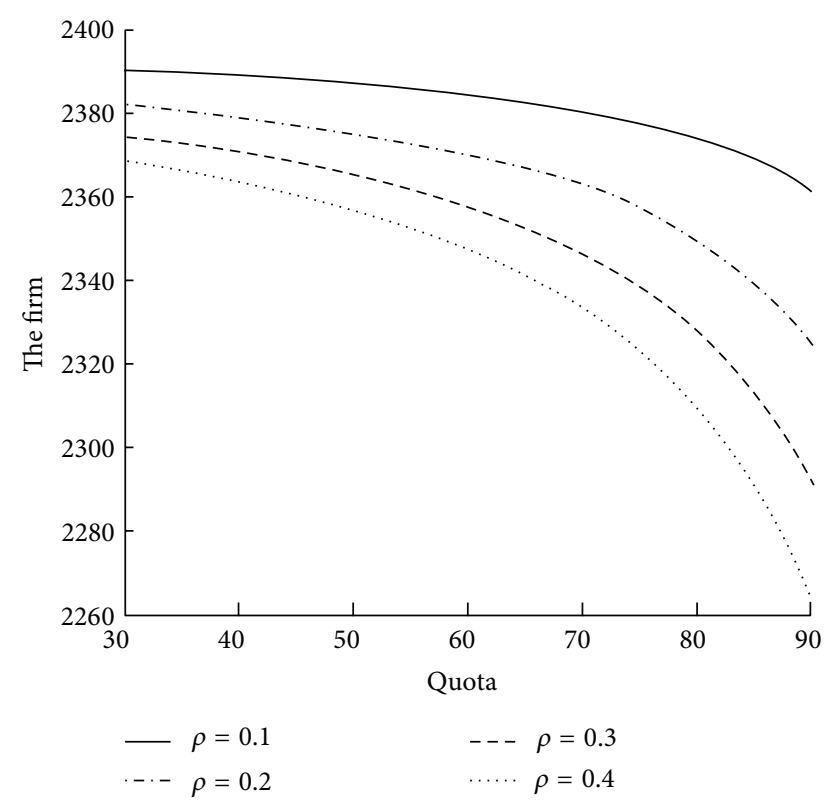

(a)

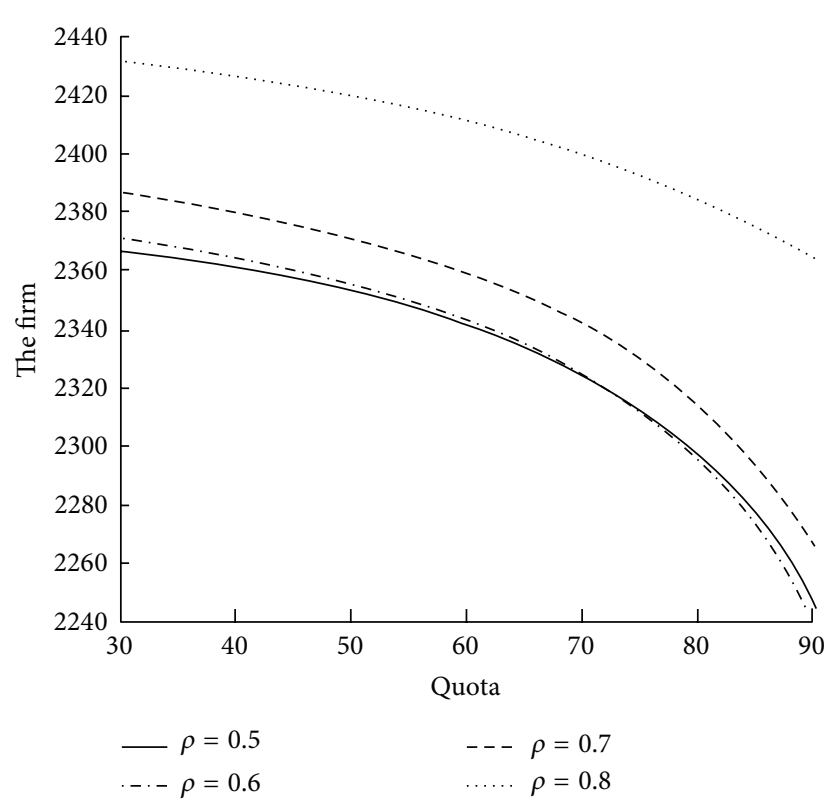

(b)

FIgURE 2: The fashion retailer's expected profit changes as the quota increases.

$Z=50$. As our goals are to study the effects of the quota $q$ and the high state probability $\rho$ on the optimal profits of the fashion retailer and the salesperson, we let $q$ vary from 30 to 90 and $\rho=\{0.1,0.2,0.3,0.4,0.5,0.6,0.7,0.8\}$.

Figure 2 shows that, for a fixed $\rho$, the fashion retailer's expected profit decreases as the quota $q$ ascends, but the decreasing rate enhances gradually. In this case, if the fashion retailer is able to choose the quota, then the best response is to select the smallest $q$ in her consideration set. Moreover, Figure 2 also indicates that, for a fixed $q$, the fashion retailer's expected profit declines gradually as $\rho$ is smaller than 0.5 while it increases as $\rho$ exceeds 0.5 .

Figure 3 implies that, for a fixed $\rho$, the fashion sales representative's optimal profit in a high state is increasing in $q$ and, in addition, the increasing rate enhances gradually. In this situation, with a larger $q$ the fashion sales representative is motivated to work hard and generate a higher sales volume, which indicates a higher profit. However, as $\rho$ approaches 1 , the fashion sales representative's optimal profit in a high state gradually reduces as $q$ increases. Moreover, Figure 3 also shows that, for a fixed $q$, the fashion sales representative's optimal profit in a higher state declines as $\rho$ ascends.

From the above numerical results, one quick implication is that a higher quota level is disadvantageous to the fashion retailer but advantageous to the fashion sales representatives. Moreover, as the high state probability increases, the numerical studies indicate that both the fashion retailer and the salespersons' optimal profits do not change monotonically.

\section{Conclusion and Remarks}

In this paper, we study the optimal compensation plan in a context of one fashion retailer and a salesperson under asym- 


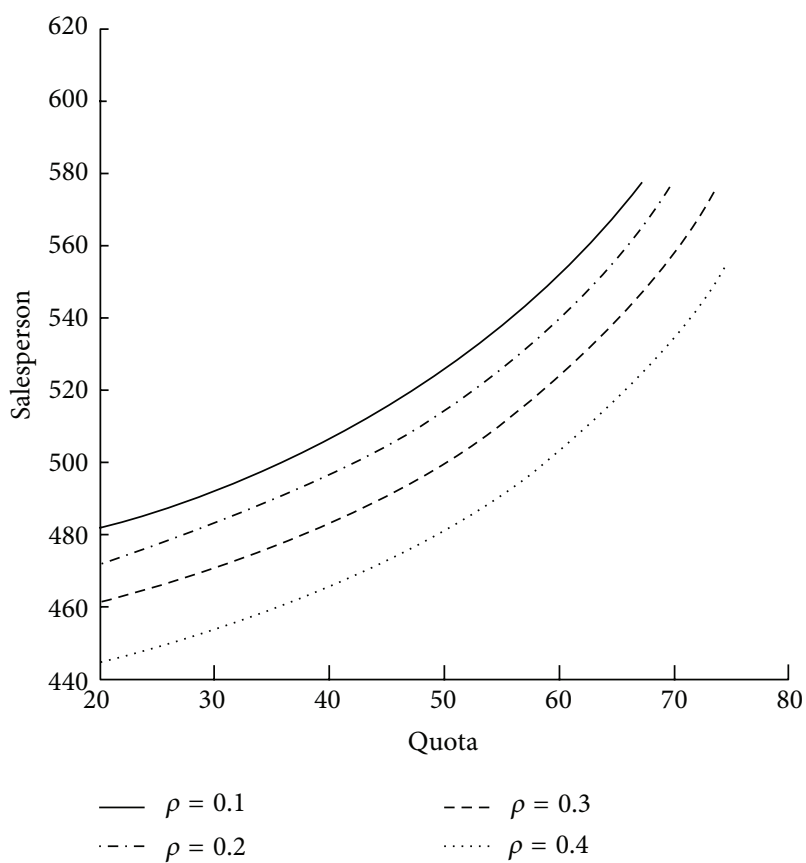

(a)

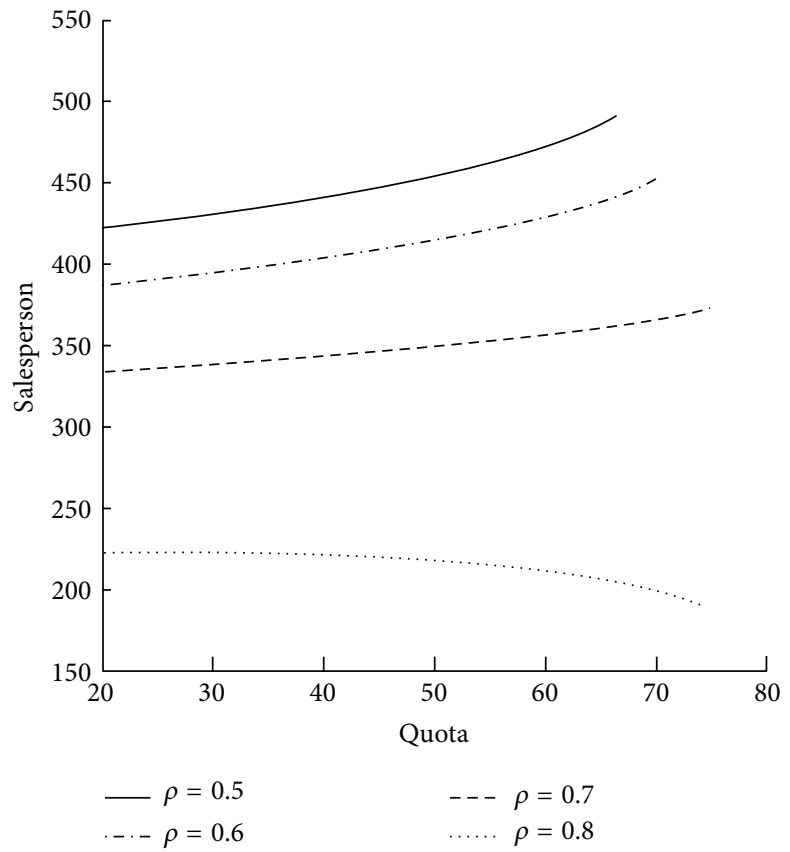

(b)

Figure 3: The salesperson's optimal profit in a high state changes as the quota increases.

metric information. We adopt the two-stage game model to analyze the problem. The quota-based compensation plan is proposed. We first derive the salesperson's optimal strategies and then formulate the design of rewarding scheme for the fashion retailer. By characterizing the optimal parameters of the quota-based compensation plan, we find that a structure of a lower fixed salary and a higher commission rate is appreciated by the fashion retailer.

In the paper, we only study a quota-based compensation plan and it is still valuable to further consider other compensation schemes. Secondly, the current paper assumes that both the fashion retailer and the salesperson are riskneutral. One useful extension is to consider the case that the fashion retailer and the salespersons are risk-averse. Finally, in the paper we discuss a compensation plan with one fashion retailer and one salesperson. However, the configuration of one fashion retailer and multiple salespersons will be valued when the competition between salespersons is emphasized. Obviously, this configuration will lead to significantly harder research and some interesting future research topics.

\section{Appendix}

Proof of Lemma 1. When the state is $\theta$, where $\theta=H$ or $L$, the sales response function of the salesperson will be $D_{\theta}=$ $d_{\theta}+\mu e+\varepsilon$.

As $\varepsilon \sim U[-Z, Z]$, we get $D_{\theta} \sim U\left[d_{\theta}+\mu e-Z, d_{\theta}+\mu e+Z\right]$, and the density function of $D_{\theta}$ is $f\left(x_{\theta}\right)=1 / 2 Z$.
Next, given the bundle $B_{\tau}$, we substitute the sales response function and the payment function in (3) into the salesperson's profit function, and then

$$
\begin{aligned}
\pi_{\theta}\left(B_{\tau}\right) & =E\left[T_{\tau}\left(D_{\theta}\right)-V(e)\right] \\
& =E\left[f_{\tau}+r_{\tau}\left(D_{\theta}-q\right)^{+}-\frac{1}{2} e^{2}\right] .
\end{aligned}
$$

Obviously, with a quota-based compensation plan, we can write out salesperson's problem as follows:

$$
\begin{aligned}
\max _{e} E & {\left[f_{\tau}+r_{\tau}\left(D_{\theta}-q\right)^{+}-\frac{1}{2} e^{2}\right] } \\
& =\max _{e}\left[\int_{q}^{d_{\theta}+\mu e+Z} r_{\tau}(x-q) \frac{1}{2 Z} d x+f_{\tau}-\frac{1}{2} e^{2}\right] \\
& =\max _{e}\left[\frac{r_{\tau}\left(d_{\theta}+\mu e+Z-q\right)^{2}}{4 Z}+f_{\tau}-\frac{1}{2} e^{2}\right] .
\end{aligned}
$$

By solving the above problem, we can derive the optimal response of the salesperson's effort level. Clearly, we claim that the above problem is concave in $e$. Then the best response of the salesperson's effort level is given by the first-order condition:

$$
\frac{r_{\tau} \mu\left(d_{\theta}+Z-q\right)}{2 Z-r_{\tau} \mu^{2}}-e=0
$$


Thus, the optimal response of salesperson's effort level under a quota-based compensation plan is given by

$$
e^{*}\left(B_{\tau}\right)=\frac{r_{\tau} \mu\left(d_{\theta}+Z-q\right)}{2 Z-r_{\tau} \mu^{2}} .
$$

Proof of Theorem 2. The optimal effort level in a high and a low state is given by

$$
\begin{gathered}
e_{L}^{*}=e\left(B_{L}(\cdot), L\right)=\frac{r_{L} \mu\left(d_{L}+Z-q\right)}{2 Z-r_{L} \mu^{2}}, \\
e_{H}^{*}=e\left(B_{H}(\cdot), H\right)=\frac{r_{H} \mu\left(d_{H}+Z-q\right)}{2 Z-r_{H} \mu^{2}} .
\end{gathered}
$$

Then we substitute the optimal effort level into problem (10), and the fashion retailer's optimization problem can be simplified by

$$
\begin{array}{cl}
\max _{B_{H}(\cdot), B_{L}(\cdot)} \quad \begin{array}{l}
\left\{\rho\left[(p-c) D_{H}-S_{H}\left(D_{H}\right)\right]\right. \\
\\
\text { s.t. }
\end{array} & \left\{\begin{array}{l}
\frac{\left.r_{L}\left(d_{L}+Z-q\right)\left[(p-c) D_{L}-S_{L}\left(D_{L}\right)\right]\right\}}{2\left(2 Z-r_{L} \mu^{2}\right)}+f_{L} \geq \pi_{\min }, \\
\frac{r_{H}\left(d_{H}+Z-q\right)^{2}}{2\left(2 Z-r_{H} \mu^{2}\right)}+f_{H} \geq \pi_{\min }, \\
\frac{r_{L}\left(d_{L}+Z-q\right)^{2}}{2\left(2 Z-r_{L} \mu^{2}\right)}+f_{L} \\
\geq \frac{r_{H}\left(d_{L}+Z-q\right)^{2}}{2\left(2 Z-r_{H} \mu^{2}\right)}+f_{H}, \\
\frac{r_{H}\left(d_{H}+Z-q\right)^{2}}{2\left(2 Z-r_{H} \mu^{2}\right)}+f_{H} \\
\geq \frac{r_{L}\left(d_{H}+Z-q\right)^{2}}{2\left(2 Z-r_{L} \mu^{2}\right)}+f_{L} .
\end{array}\right.
\end{array}
$$

Next, we claim that the first and the last constraints are binding as long as $r_{H} \geq r_{L}$.

First of all, if the first and the fourth constraints hold, then the second constraint holds automatically. Notice that, from the first constraint, we have $f_{L} \geq \pi_{\min }-\left(r_{L}\left(d_{L}+Z-q\right)^{2} / 2(2 Z-\right.$ $\left.\left.r_{L} \mu^{2}\right)\right)$. Replace it into the last constraint; since $d_{H}>d_{L}$, then we get

$$
\begin{aligned}
\frac{r_{H}\left(d_{H}+Z-q\right)^{2}}{2\left(2 Z-r_{H} \mu^{2}\right)}+f_{H} \geq & \pi_{\min }+\frac{r_{L}\left(d_{H} Z-q\right)^{2}}{2\left(2 Z-r_{L} \mu^{2}\right)} \\
& -\frac{r_{L}\left(d_{L}+Z-q\right)^{2}}{2\left(2 Z-r_{L} \mu^{2}\right)} \geq \pi_{\min } .
\end{aligned}
$$

Secondly, we show that the first constraint is binding. If not, we can decrease $f_{L}$ and $f_{H}$ by the same amount $\varepsilon$ such that all the constraints still hold and the objective function will increase, which violates the optimality condition.

Thirdly, we show that the last constraint is binding as long as $r_{H} \geq r_{L}$. If, at the optimal solution, the last constraint is not binding, then we can decrease $f_{H}$ by $\varepsilon$ such that the last constraint is binding. In this case, all other constraints still hold and the objective function will increase, which violates the optimality condition.

Hence, as long as $r_{H} \geq r_{L}$, the third constraint holds automatically. When the first and the last constraints are tight, we get

$$
\begin{aligned}
& f_{L}=\pi_{\min }-\frac{r_{L}\left(d_{L}+Z-q\right)^{2}}{2\left(2 Z-r_{L} \mu^{2}\right)} ; \\
& f_{H}=f_{L}+\frac{r_{L}\left(d_{H}+Z-q\right)^{2}}{2\left(2 Z-r_{L} \mu^{2}\right)}-\frac{r_{H}\left(d_{H}+Z-q\right)^{2}}{2\left(2 Z-r_{H} \mu^{2}\right)} .
\end{aligned}
$$

Substituting $f_{L}$ and $f_{H}$ into the third constraint, we get

$$
\begin{gathered}
\frac{r_{L}\left(d_{L}+Z-q\right)^{2}}{2\left(2 Z-r_{L} \mu^{2}\right)}+f_{L}-\frac{r_{H}\left(d_{L}+Z-q_{i}\right)^{2}}{2\left(2 Z-r_{H} \mu^{2}\right)}+f_{H} \\
=\frac{r_{L}\left(d_{L}+Z-q\right)^{2}}{2\left(2 Z-r_{L} \mu^{2}\right)}-\frac{r_{H}\left(d_{L}+Z-q_{i}\right)^{2}}{2\left(2 Z-r_{H} \mu^{2}\right)} \\
\quad-\frac{r_{L}\left(d_{H}+Z-q\right)^{2}}{2\left(2 Z-r_{L} \mu^{2}\right)}+\frac{r_{H}\left(d_{H}+Z-q\right)^{2}}{2\left(2 Z-r_{H} \mu^{2}\right)} \\
=-\frac{r_{L}\left(d_{H}+d_{L}+2 Z-2 q\right)\left(d_{H}-d_{L}\right)}{2\left(2 Z-r_{L} \mu^{2}\right)} \\
+\frac{r_{H}\left(d_{H}+d_{L}+2 Z-2 q\right)\left(d_{H}-d_{L}\right)}{2\left(2 Z-r_{H} \mu^{2}\right)} \geq 0 .
\end{gathered}
$$

From all the above, we get that the first and the fourth constraints are binding as long as $r_{H} \geq r_{L}$.

Subsequently, we turn to analyze the optimal commission rate and the fixed salary under a quota-based compensation plan. Relaxing the second and the third constraints, we can convert the original constrained optimization problem into an unconstrained optimization problem. We substitute the optimal effort levels $f_{L}$ and $f_{H}$ into the objective function, and then the fashion retailer's optimization problem can be written as

$\Pi$

$$
\begin{aligned}
= & \rho\left[(p-c)\left(d_{H}+\mu e_{H}^{*}\right)-\frac{r_{H}\left(d_{H}+\mu e_{H}^{*}+Z-q\right)^{2}}{4 Z}-f_{H}\right] \\
& +(1-\rho) \\
& \times\left[(p-c)\left(d_{L}+\mu e_{L}^{*}\right)-\frac{r_{L}\left(d_{L}+\mu e_{L}^{*}+Z-q\right)^{2}}{4 Z}-f_{L}\right]
\end{aligned}
$$




$$
\begin{aligned}
= & \rho\left[(p-c) \frac{2 d_{H} Z+r_{H} \mu^{2}(Z-q)}{2 Z-r_{H} \mu^{2}}-\frac{r_{H}^{2} \mu^{2}\left(d_{H}+Z-q\right)^{2}}{2\left(2 Z-r_{H} \mu^{2}\right)^{2}}\right] \\
& -\frac{\rho r_{L}\left(d_{H}+d_{L}+2 Z-2 q\right)\left(d_{H}-d_{L}\right)}{2\left(2 Z-r_{L} \mu^{2}\right)} \\
& +(1-\rho) \\
& \times\left[(p-c) \frac{2 d_{L} Z+r_{L} \mu^{2}(Z-q)}{2 Z-r_{L} \mu^{2}}-\frac{r_{L}^{2} \mu^{2}\left(d_{L}+Z-q\right)^{2}}{2\left(2 Z-r_{L} \mu^{2}\right)^{2}}\right] .
\end{aligned}
$$

Note that the fashion retailer's objective function is separable and concave in $r_{H}$ and $r_{L}$, respectively. Thus, the optimal commission rate can be derived by the first-order condition.

Denote $A_{H}^{0}=r_{H} /\left(2 Z-r_{H} \mu^{2}\right)$ and $A_{L}^{0}=r_{L} /\left(2 Z-r_{L} \mu^{2}\right)$. And $\partial A_{H}^{0} / \partial r_{H}=2 Z /\left(2 Z-r_{H} \mu^{2}\right)^{2}$ and $\partial A_{L}^{0} / \partial \alpha_{i L}=2 Z /(2 Z-$ $\left.r_{L} \mu^{2}\right)^{2}$. In this case, the fashion retailer's objective function can be written as

$$
\begin{gathered}
\Pi=\rho\left[\frac{2(p-c) d_{H} Z}{2 Z-r_{H} \mu^{2}}+A_{H}^{0}(p-c) \mu^{2}(Z-q)\right. \\
\left.-\left(A_{H}^{0}\right)^{2} \frac{\mu^{2}\left(d_{H}+Z-q\right)^{2}}{2}\right] \\
-A_{L}^{0} \frac{\rho\left(d_{H}+d_{L}+2 Z-2 q\right)\left(d_{H}-d_{L}\right)}{2} \\
+(1-\rho)\left[\frac{2(p-c) d_{L} Z}{2 Z-r_{L} \mu^{2}}+A_{L}^{0}(p-c) \mu^{2}(Z-q)\right. \\
\left.-\left(A_{L}^{0}\right)^{2} \frac{\mu^{2}\left(d_{L}+Z-q\right)^{2}}{2}\right] .
\end{gathered}
$$

Then we take the first derivative with respect to $r_{H}$ and set it to 0 . Consider

$$
\begin{aligned}
\frac{\partial \Pi}{\partial r_{H}}= & \frac{2 \mu^{2}(p-c) d_{H} Z}{\left(2 Z-r_{H} \mu^{2}\right)^{2}}+\frac{\partial A_{H}^{0}}{\partial r_{H}}(p-c) \mu^{2}(Z-q) \\
& -A_{H}^{0} \frac{\partial A_{H}^{0}}{\partial r_{H}} \mu^{2}\left(d_{H}+Z-q\right)^{2} \\
= & \frac{2 \mu^{2} Z\left(d_{H}+Z-q\right)}{\left(2 Z-r_{H} \mu^{2}\right)^{2}}\left[p-c-A_{H}^{0}\left(d_{H}+Z-q\right)\right]=0 .
\end{aligned}
$$

Thus, the optimal commission rate in a high state $r_{H}$ is given by

$$
r_{H}=\frac{2(p-c) Z}{d_{H}+Z-q+\mu^{2}(p-c)}
$$

Similarly, we can drive the optimal commission rate in a low state, since

$$
\begin{aligned}
\frac{\partial \Pi}{\partial r_{L}}= & \frac{2(1-\rho) \mu^{2} Z\left(d_{L}+Z-q\right)\left[p-c-A_{L}^{0}\left(d_{L}+Z-q\right)\right]}{\left(2 Z-r_{L} \mu^{2}\right)^{2}} \\
& -\frac{\partial A_{L}^{0}}{\partial r_{L}} \frac{\rho\left(d_{H}+d_{L}+2 Z-2 q\right)\left(d_{H}-d_{L}\right)}{2} \\
= & \left(\left(2(1-\rho) \mu^{2} Z\left(d_{L}+Z-q\right)\right.\right. \\
& \left.\times\left[p-c-\frac{r_{L}}{2 Z-r_{L} \mu^{2}}\left(d_{L}+Z-q\right)\right]\right) \\
& \left.\times\left(2 Z-r_{L} \mu^{2}\right)^{-2}\right) \\
& \frac{\rho Z\left(d_{H}+d_{L}+2 Z-2 q\right)\left(d_{H}-d_{L}\right)}{\left(2 Z-r_{L} \mu^{2}\right)^{2}}=0 .
\end{aligned}
$$

As $r_{L} \geq 0$, thus $r_{L}$ is given by

$$
\begin{aligned}
r_{L}=\max \{0, & \left(2 Z\left[2(1-\rho) \mu^{2}(p-c) T_{L}-\rho\left(T_{L}+T_{H}\right) I\right]\right) \\
\times( & \left(2(1-\rho) \mu^{2} T_{L}^{2}+2(1-\rho) \mu^{4}(p-c) T_{L}\right. \\
& \left.\left.-\rho \mu^{2}\left(T_{L}+T_{H}\right) I\right)^{-1}\right\} .
\end{aligned}
$$

Finally, we turn to testify the following condition: $r_{H} \geq r_{L}$. Consider

$$
\begin{aligned}
& \left(\left(2 Z \left[2(1-\rho) \mu^{2}(p-c) T_{L}\right.\right.\right. \\
& \left.\left.-\rho\left(T_{L}+T_{H}\right)\left(d_{H}-d_{L}\right)\right]\right) \\
& \times\left(2(1-\rho) \mu^{2} T_{L}^{2}+2(1-\rho) \mu^{4}(p-c) T_{L}\right. \\
& \left.\left.\quad-\rho \mu^{2}\left(T_{L}+T_{H}\right)\left(d_{H}-d_{L}\right)\right)^{-1}\right) \\
& \leq((2 Z(p-c)) \\
& \quad \times\left(2(1-\rho) \mu^{2} T_{L}^{2}+2(1-\rho) \mu^{4}(p-c) T_{L}\right. \\
& \left.\left.\quad-\rho \mu^{2}\left(T_{L}+T_{H}\right)\left(d_{H}-d_{L}\right)\right)^{-1}\right) \\
& \leq \frac{2(p-c) Z}{T_{H}+\mu^{2}(p-c)}=r_{H} .
\end{aligned}
$$

From all the above, we can summarize the following.

When the two salespersons are independent, the optimal parameters of a quota-based plan are given by the following. 
(i) The optimal commission rate is as follows:

$$
\begin{gathered}
r_{L} \\
=\max \left\{0,\left(2 Z\left[2(1-\rho) \mu^{2}(p-c) T_{L}-\rho\left(T_{L}+T_{H}\right) I\right]\right)\right. \\
\times\left(2(1-\rho) \mu^{2} T_{L}^{2}+2(1-\rho) \mu^{4}(p-c) T_{L}\right. \\
\left.\left.-\rho \mu^{2}\left(T_{L}+T_{H}\right) I\right)^{-1}\right\} \\
r_{H}=\frac{2(p-c) Z}{T_{H}+\mu^{2}(p-c)} .
\end{gathered}
$$

(ii) And the optimal fixed salary is as follows:

$$
\begin{gathered}
f_{L}=\pi_{\min }-\frac{r_{L}\left(d_{L}+Z-q\right)^{2}}{2\left(2 Z-r_{L} \mu^{2}\right)} ; \\
f_{H}=f_{L}+\frac{r_{L}\left(d_{H}+Z-q\right)^{2}}{2\left(2 Z-r_{L} \mu^{2}\right)}-\frac{r_{H}\left(d_{H}+Z-q\right)^{2}}{2\left(2 Z-r_{H} \mu^{2}\right)} .
\end{gathered}
$$

Here, $T_{L}=d_{L}+Z-q, T_{H}=d_{H}+Z-q, T=T_{H}+T_{L}$, and $I=d_{H}-d_{L}$.

Proof of Proposition 3. From Theorem 2, we get $r_{H}^{0}=2(p-$ c) $Z /\left(r_{H}+Z-q+\mu^{2}(p-c)\right)$. Obviously, $r_{H}$ is increasing in $q$.

By Theorem 2, we have

$$
\begin{aligned}
& r_{L}=(\left(2 Z\left[2(1-\rho) \mu^{2}(p-c) T_{L}-\rho\left(T_{L}+T_{H}\right) I\right]\right) \\
& \times\left(2(1-\rho) \mu^{2} T_{L}^{2}\right. \\
&\left.\left.\quad+2(1-\rho) \mu^{4}(p-c) T_{L}-\rho \mu^{2}\left(T_{L}+T_{H}\right) I\right)^{-1}\right) .
\end{aligned}
$$

Denote $\Delta=2(1-\rho) \mu^{2} T_{L}^{2}+2(1-\rho) \mu^{4}(p-c) T_{L}-\rho \mu^{2}\left(T_{L}+T_{H}\right) I$. Then we take the first derivative with respect to $q$. Consider

$$
\begin{aligned}
\frac{\partial r_{L}}{\partial q}= & \frac{1}{\Delta^{2}} 8 Z \mu^{2}(1-\rho)\left(d_{L}+Z-1\right) \\
& \times\left[\mu^{2}(1-\rho)(p-c)\left(d_{L}+Z-q\right)\right. \\
& \left.-\rho\left(d_{H}+Z-q\right)\left(d_{H}-d_{L}\right)\right] .
\end{aligned}
$$

Thus $\partial r_{L} / \partial q \geq 0$ if and only if $(1-\rho)\left(d_{L}+Z-q\right) / \rho\left(d_{H}+Z-q\right) \geq$ $\left(d_{H}-d_{L}\right) / \mu^{2}(p-c)$.
For the optimal fixed salary, we have

$$
\begin{aligned}
\frac{\partial f_{L}}{\partial q}= & \frac{d_{L}+Z-q}{2 Z-r_{L}^{0} \mu^{2}}\left[r_{L}^{0}-\frac{Z\left(d_{i L}+Z-q\right)}{2 Z-r_{L}^{0} \mu^{2}} \frac{\partial r_{L}^{0}}{\partial q}\right] ; \\
\frac{\partial f_{H}}{\partial q}= & \frac{Z\left[\left(d_{H}+Z-q\right)^{2}-\left(d_{L}+Z-q\right)^{2}\right]}{\left(2 Z-r_{L} \mu^{2}\right)^{2}} \frac{\partial r_{L}}{\partial q} \\
& -\frac{Z\left(d_{H}+Z-q\right)^{2}}{\left(2 Z-r_{H}^{0} \mu^{2}\right)^{2}} \frac{\partial r_{H}}{\partial q}+\frac{r_{H}\left(d_{H}+Z-q\right)}{2 Z-r_{H}^{0} \mu^{2}} \\
& -\frac{r_{L}\left(d_{H}-d_{L}\right)}{2 Z-r_{L} \mu^{2}} .
\end{aligned}
$$

Obviously, if $(1-\rho)\left(d_{L}+Z-q\right) / \rho\left(d_{H}+Z-q\right)<\left(d_{H}-\right.$ $\left.d_{L}\right) / \mu^{2}(p-c)$, we have $\partial f_{L} / \partial q>0$ and $\partial f_{H} / \partial q>0$.

From all the above, we summarize the following. (i) $r_{H}$ ascends as the quota $q$ increases, while $r_{H}$ is increasing in $q$ if and only if $(1-\rho)\left(d_{L}+Z-q\right) / \rho\left(d_{H}+Z-q\right) \geq\left(d_{H}-d_{L}\right) / \mu^{2}(p-$ $c)$. (ii) Moreover, if $(1-\rho)\left(d_{L}+Z-q\right) / \rho\left(d_{H}+Z-q\right)<$ $\left(d_{H}-d_{L}\right) / \mu^{2}(p-c)$, both $f_{L}$ and $f_{H}$ increase as $q$ rises.

\section{Conflict of Interests}

The authors declare that there is no conflict of interests regarding the publication of this paper.

\section{Acknowledgments}

This research was partially supported by the CPSF Grant no. 2012M521784, the National Natural Science Fund of China (NSFC) nos. 71001073 and 71302109, and the Distinguished University Young Scholar Program of Guangdong Province no. Yq2013140.

\section{References}

[1] D. Kreps, A Course in Microeconomic Theory, Princeton University Press, Princeton, NJ, USA, 1990.

[2] A. Coughlan and S. Sen, "Salesforce compensation: theory and managerial implications," Marketing Science, vol. 8, no. 4, pp. 324-342, 1989.

[3] A. Coughlan, "Salesforce compensation: a review of MS/OR advances," in Handbooks in Operations Research and Management Science: Marketing, J. Eliashberg and G. Lilien, Eds., vol. 5, North-Holland, Amsterdam, The Netherlands, 1993.

[4] J. Farley, “An optimal plan for salesmen's compensation,” Journal of Marketing Research, vol. 1, no. 2, pp. 39-43, 1964.

[5] C. Tapiero and J. Farley, "Optimal control of salesforce effort in time," Management Science, vol. 21, no. 9, pp. 976-985, 1975.

[6] C. Weinberg, "Jointly optimal sales commissions for nonincome maximizing salesforce," Management Science, vol. 24, no. 12, pp. 1252-1258, 1978.

[7] A. Basu, R. Lal, V. Srinivasan, and R. Staelin, "Salesforce compensation plans: an agency theoretic perspective," Marketing Science, vol. 4, no. 4, pp. 267-291, 1985.

[8] V. Srinivasan, "An investigation of the equal commission rate policy for a multi-product salesforce," Management Science, vol. 27, no. 7, pp. 731-756, 1981. 
[9] R. Lal and V. Srinivasan, "Compensation plans for single- and multi-product salesforces: an application of the holmstrommilgrom model," Management Science, vol. 39, no. 7, pp. 777793, 1993.

[10] R. Lal, "Delegating pricing responsibility to the salesforce," Marketing Science, vol. 5, no. 2, pp. 159-168, 1986.

[11] R. Lal and R. Staelin, "Salesfoce-compensation plans in environments with asymmetric information," Marketing Science, vol. 5, no. 3, pp. 179-198, 1986.

[12] J. Dearden and G. Lilien, "On optimal salesforce compensation in the presence of production learning effects," International Journal of Research in Marketing, vol. 7, no. 2-3, pp. 179-188, 1990.

[13] R. Rao, "Compensating heterogeneous salesforces: some explicit solutions," Marketing Science, vol. 9, no. 4, pp. 319-341, 1990.

[14] F. Chen, "Salesforce incentives, market information, and production/inventory planning," Management Science, vol. 51, no. 1, pp. 60-75, 2005.

[15] F. Chen, "Provision of incentives for information acquisition: a comparison between forecast-based contracts and menus of linear contracts," Working Paper, Columbia Business School, Manhattan, NY, USA, 2009.

[16] F. Chen, "Sales-force incentives and inventory management," Manufacturing and Service Operations Management, vol. 2, no. 2, pp. 186-202, 2000.

[17] E. Porteus and S. Whang, "On manufacturing/marketing incentives," Management Science, vol. 37, no. 9, pp. 1166-1181, 1991.

[18] M. K. Mantrala, K. Raman, and R. Desiraju, "Optimal sales quota-setting with bayesian learning of salesperson selling effectiveness by the fashion retailer," Working Paper, University of Florida, 1993.

[19] J. S. Raju and V. Srinivasan, "Quota-based compensation plans for multiterritory heterogeneous salesforces," Management Science, vol. 42, no. 10, pp. 1454-1462, 1996.

[20] C.-H. Chiu, T.-M. Choi, H.-T. Yeung, and Y. Zhao, "Sales rebate contracts in fashion supply chains," Mathematical Problems in Engineering, vol. 2012, Article ID 908408, 19 pages, 2012.

[21] H. Kurata and X. Yue, "Trade promotion mode choice and information sharing in fashion retail supply chains," International Journal of Production Economics, vol. 114, no. 2, pp. 507-519, 2008.

[22] M. Xu, Q. Wang, and L. Ouyang, "Coordinating contracts for two-stage fashion supply chain with risk-averse retailer and price-dependent demand," Mathematical Problems in Engineering, vol. 2013, Article ID 259164, 12 pages, 2013.

[23] Y. Wang, B. Niu, and P. Guo, "The comparison of two vertical outsourcing structures under push and pull contracts," Production and Operations Management, vol. 23, no. 4, pp. 610-625, 2014.

[24] A. Y. Ha and S. Tong, "Contracting and information sharing under supply chain competition," Management Science, vol. 54, no. 4, pp. 701-715, 2008.

[25] H. Li, P. Ritchken, and Y. Wang, "Option and forward contracting with asymmetric information: valuation issues in supply chains," European Journal of Operational Research, vol. 197, no. 1, pp. 134-148, 2009.

[26] C.-Y. Lee and R. Yang, "Supply chain contracting with competing suppliers under asymmetric information," IIE Transactions, vol. 45, no. 1, pp. 25-52, 2013. 


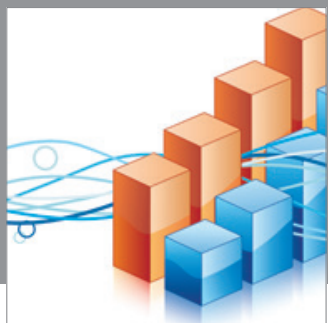

Advances in

Operations Research

mansans

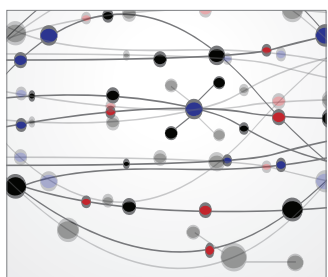

The Scientific World Journal
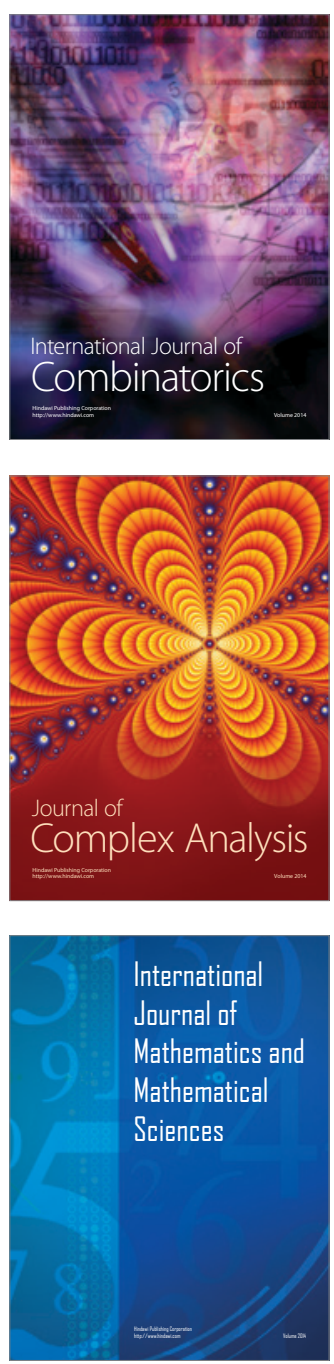
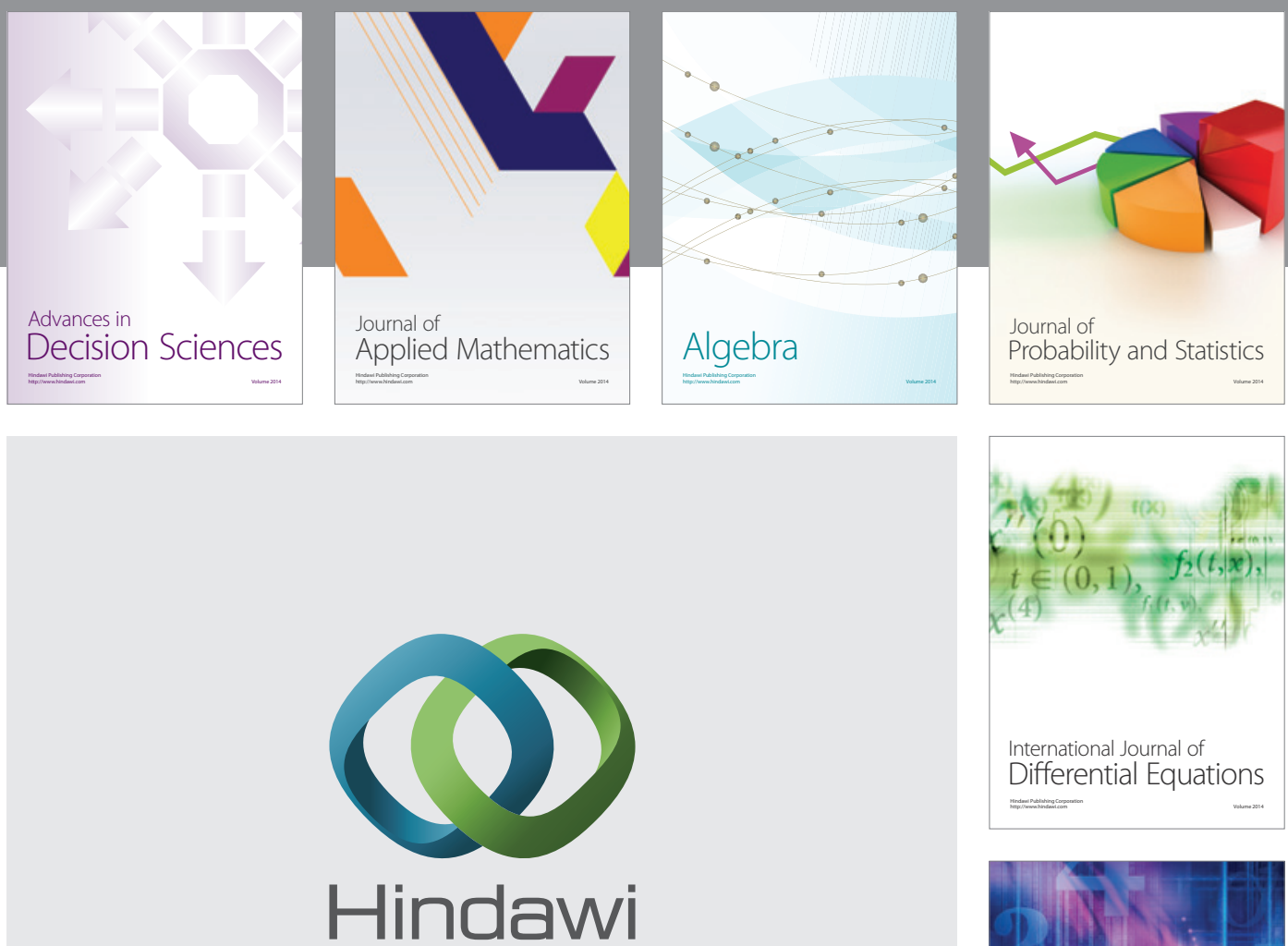

Submit your manuscripts at http://www.hindawi.com
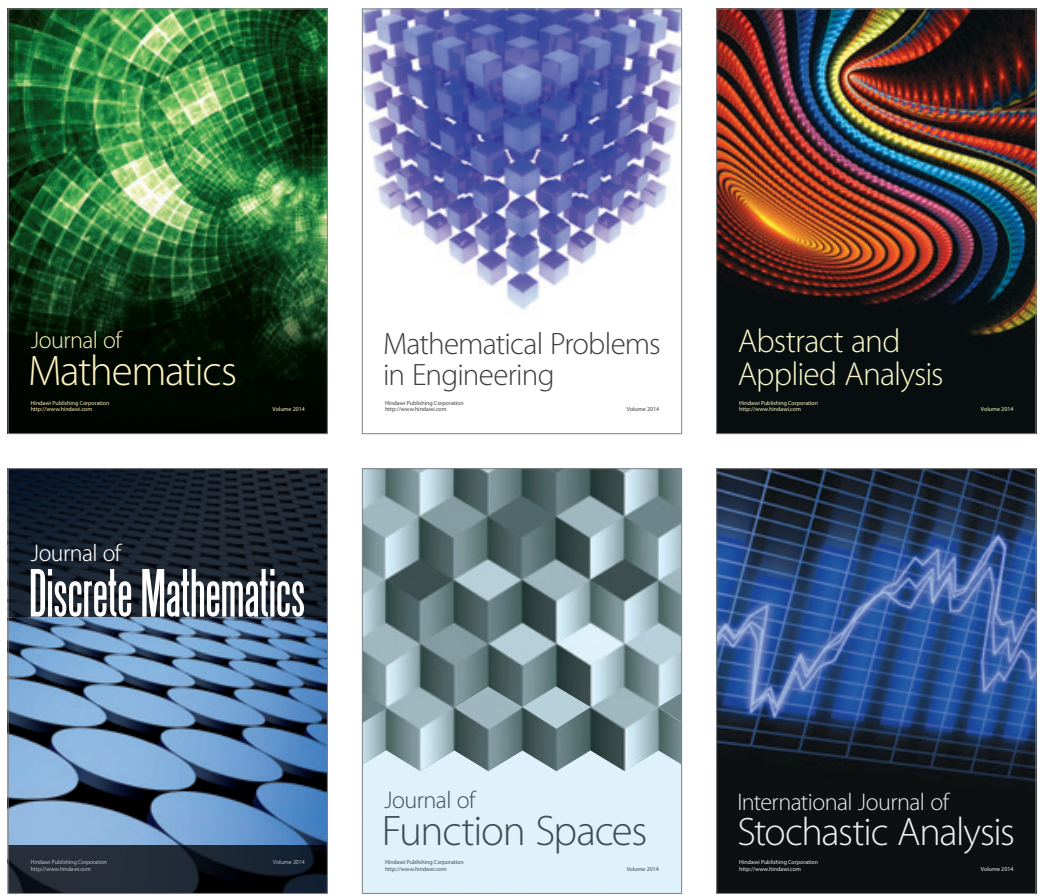

Journal of

Function Spaces

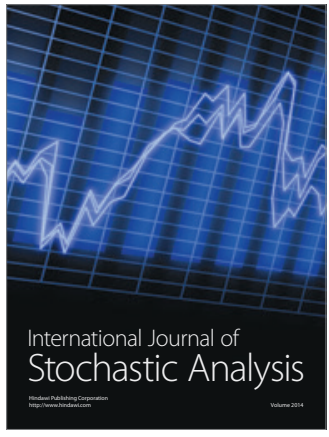

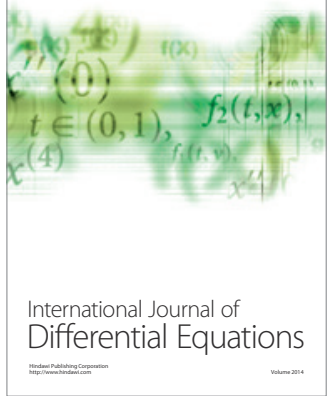
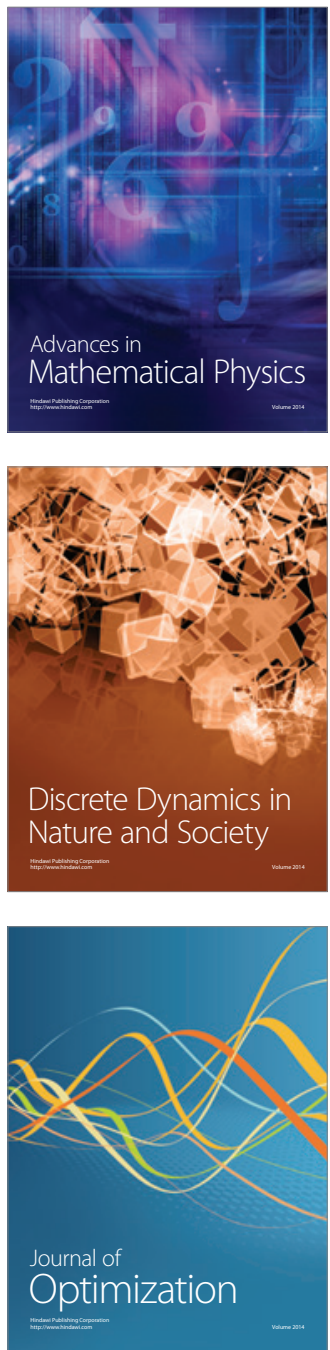